\title{
Identification of prognostic biomarkers for response to radiotherapy by DNA microarray in nasopharyngeal carcinoma patients
}

\author{
SHU YANG ${ }^{1 *}$, JIAXIN CHEN $^{2 *}$, YI GUO $^{1}$, HUI LIN $^{2}$, ZHENG ZHANG $^{2}$, GUOSHENG FENG $^{2}$,

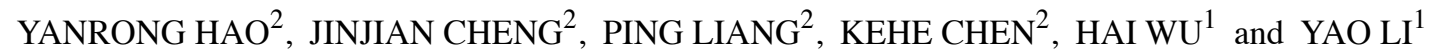 \\ ${ }^{1}$ Life State Key Laboratory of Genetic Engineering, Institute of Genetics, School of Life Science, \\ Fudan University, Shanghai; ${ }^{2}$ Institute of Nasopharyngeal Carcinoma, The People's Hospital \\ of Guangxi Zhuang Nationality Autonomous Region, Nanning, P.R. China
}

Received November 3, 2011; Accepted December 28, 2011

DOI: $10.3892 /$ ijo.2012.1341

\begin{abstract}
Nasopharyngeal carcinoma (NPC) is a malignancy with an unusually variable incidence rate across the world. Radiotherapy is the primary treatment modality for NPC, but radiation resistance remains a serious obstacle to successful treatment in many cases. To identify the genes involved in this resistance and to find molecular markers for predicting NPC response to radiotherapy, we compare the expression profiles of 12 radiation-resistant patient biopsy specimens and 8 radiationsensitive patient biopsy specimens using DNA microarray, containing 14112 human unigenes. A total of 111 aberrantly expressed genes were identified, of which ZNF608 and CSF1R were up-regulated in the radiation-resistant NPC compared with radiation-sensitive NPC, and the results were confirmed by real-time RT-PCR in 17 independent NPC patient specimens. Biostatistics and bioinformatics analyses were performed to detect the potential pathway underling this resistance, 26 pathways ( 9 categories) were found probably associated with radiation-resistant NPC, such as cell ion homeostasis, cell proliferation, receptor protein signalling, membrane system, humoral immune response, as well as cytokines and inflammation. We suggest the radiation-resistant capacity of NPC was mostly due to the change of cell $\mathrm{Ca}^{2+}$ homeostasis promoting anti-apoptosis, DNA repair and rescuing tumour cells under radiation therapy. Cell proliferation promotion induced by extracellular and intra-
\end{abstract}

Correspondence to: Professor Yao Li, Life State Key Laboratory of Genetic Engineering, Institute of Genetics, School of Life Science, Fudan University, Shanghai, P.R. China

E-mail: yaoli@fudan.edu.cn

Professor Jiaxin Chen, Institute of Nasopharyngeal Carcinoma, The People's Hospital of Guangxi Zhuang Nationality Autonomous Region, Nanning, P.R. China

E-mail: cjx166@yahoo.com.cn

*Contributed equally

Key words: DNA microarray, nasopharyngeal carcinoma, radiotherapy, radiation-resistant, differentially expressed gene, pathway enrichment cellular factors may maintain tumour size under radiotherapy leading to recurrence after treatment. Our study reveals that at least 2 ectopically expressed genes play important roles in prognosis of NPC radiotherapy and may serve as potential targets for novel radiation therapeutic strategies in the future.

\section{Introduction}

Nasopharyngeal carcinoma (NPC) is a non-lymphomatous, squamous cell malignancy arising from the epithelial cell lining of the nasopharynx (1). Compared to other malignant tumours of the upper aero digestive tract, NPC is a special type of head and neck cancer in terms of epidemiology, pathology, and clinical presentation. The aetiology of NPC involves multiple factors including genetic susceptibility, exposure to chemical carcinogens, and Epstein-Barr virus (EBV) infection (2-5). Histologically, the World Health Organization (WHO) has classified NPC into three main types: keratinizing squamous cell carcinoma (WHO type I), differentiated non-keratinizing squamous cell carcinoma (type II) and undifferentiated carcinomas (type III) (1). In some regions, notably the southern parts of China, and parts of Southeast Asia, this cancer occurs in an endemic form with an incidence 10- to 30-fold higher than the other regions and, histologically, usually belong to WHO types II and III (6). However, in the west, NPC occurs sporadically and usually belongs to type I. There is also increased incidence in northern Africa and the Inuit of Alaska (7).

Comparing with other head and neck cancers, NPC tends to be more sensitive to ionizing radiation. Additionally, WHO types II and III are more sensitive to ionizing radiation than type I. Thus, radiotherapy is the major treatment modality for NPC, but radio-resistance remains a serious obstacle to successful treatment in many cases. The 5-year survival rate after treatment is only $50-60 \%(8,9)$. In relatively advanced disease, combined radio-chemotherapy may increase survival (10). Some of the NPC patients present local recurrences and distant metastases after radiotherapy due to radiation resistance and the majority of these patients succumb to recurrence and metastasis within 1.5 year after treatment $(11,12)$. Hence, revealing the molecular mechanism of NPC radiation resistance is urgently needed 
for personalized therapy. Although a fraction of genes such as elements of cell cycle control, apoptosis/anti-apoptosis, and DNA repair are believed to play a key role in the ionizing radiationinduced cell damage, the understanding of radiation resistance in cancer at a molecular level remains limited.

Microarray methods have been widely used to detect the difference between NPC and non-neoplastic tissues $(13,14)$, and to assess genes involved in radiation resistance in a number of cancer cell types, including cervical, pancreatic, oral, lung, oesophageal cancers (15-19). Analysis for gene expression profiles of radiation-resistant NPC cell lines using a cDNA microarray found that at least 2 genes, gp96/HSP90B1 and GDF15, involved in the radiation resistance of NPC (20). Recently, 183 differentially expressed genes and 23 biological processes were identified to be relevant to NPC radiation resistance (21). Furthermore, proteomics was then performed to compare the protein profiles of CNE2-IR and CNE2 cell lines (22). Thirty-four differential proteins were identified in the radiation-resistant $\mathrm{CNE}-2$-IR and the expression levels of four differential proteins (14-3-3o/SFN, Maspin/SERPINB5, GRP78/HSPA5, and Mn-SOD/SOD2) were confirmed. However, there was no overlap in the genes found to be involved in radiation resistance in these studies. This may be because of distinct tissue specificity, but it is also possible that there is some fundamental mechanism underlying radiation resistance that has not yet been elucidated. Qu et al (23) have demonstrated that SOD2 was over-expressed in relatively radiation-resistant NPC cell line CNE1 compared with CNE2, and knockdown of SOD2 by microRNA significantly decreased radiation resistance of CNE1 cells. Besides, the activity of DNA-dependent protein kinase was also found higher in CNE1 than CNE2 cell line (24).

Tumour cells are known to be most sensitive to radiationinduced cell death when synchronized in the $G_{2}-M$ phase of the cell cycle, as the DNA is more vulnerable to radiation-induced damage during mitosis. Most existing radiation-resistance mechanisms indicate that radiation resistance is associated with an up-regulation of DNA repair and stress response genes, down-regulation of cell cycle and apoptosis genes, and immune response to organize genes (25). Substantial relevant NPC research support the view that radiation resistance is associated with DNA damage repair, apoptosis, cell cycle checkpoints, and immune response (20-22). Moreover, Guo et al (21) found 23 biological processes involved in NPC radiation resistance, including type I interferon-mediated signalling pathway, cellular response to cytokine stimulus, response to other organisms, response to viruses, immune system process, and response to stress. They suggested that over-expression of components of type I interferon-mediated signalling pathways plays a role in radiation resistance of NPC through enhanced cell survival after radiation. However, the molecular mechanisms underlying NPC radiation resistance are still unclear, till now; there have not been effective biomarkers for predicting NPC radiation sensitivity in clinic.

Although many studies found more significant outcome of NPC radiation sensitivity by comparing radiation-resistant NPC cells with radiation-sensitive NPC cells, limited investigations have been focused on the difference between these two kinds of clinical NPC biopsy specimens. In this study, we aimed to identify important molecular markers for response to radiotherapy of poor differentiation NPC patients by DNA microarray.
Alterations in gene expression and signalling pathway patterns provide important clues on these complex biological processes. The use of DNA microarray technology provides us the opportunity to learn more about the mechanisms underlying the radiation resistance of NPC. We identified 111 genes and 9 categories of possible biological processes involved in radiation resistance of NPC patients. Two of aberrantly expressed genes, CSF1R and ZNF608, were chosen for validation by real-time RT-PCR in 17 independent NPC specimens. We expect our analysis to provide some clues to clarify the mechanism of NPC radiation resistance. In addition, we explored the difference in RRNPC and RSNPC compared with NPCI, respectively.

\section{Materials and methods}

Tissue specimens. Nasopharyngeal carcinoma (NPC) tissue samples were obtained from 37 patients with poorly differentiated squamous NPC before radiation treatment at the Institute of Nasopharyngeal Carcinoma, the People's Hospital of Guangxi Zhuang Nationality Autonomous Region, Nanning, China. Of them, 20 patient specimens were used for microarray experiments. The rest of them were used for quantitative real-time reverse transcription-polymerase chain reaction (RT-PCR). The information of NPC samples are listed in Table I. Additionally, a pool of nasopharyngeal chronic inflammation (NPCI) tissues, as common reference for microarray experiments, were obtained in the same hospital. All the specimens were reviewed by otorhinolaryngology pathologist, and with consent in accordance with approval granted by the Ethics Committee of the Institute of Nasopharyngeal Carcinoma, The People's Hospital of Guangxi Zhuang Nationality Autonomous Region. Fresh NPC and NPCI tissues were snap-frozen in liquid nitrogen and stored until use. Here, we defined the patients with $<40 \%$ reduction of tumour size after radiation treatment as radiationresistant NPC patients, and the patients with $>60 \%$ reduction of tumour size as radiation-sensitive NPC patients.

RNA isolation and reverse transcription. Total RNA was extracted from all NPC patient specimens using TRIzol (Invitrogen, Carlsbad, CA, USA) reagent according to the manufacturer's instructions. All RNA samples were examined for concentration and purity based on the agarose gel electrophoresis and absorbance ratio at 260-280 $\mathrm{nm}$ to make sure of the RNA quality. Total RNA was reverse transcribed with Oligo(dT) primers using the Superscript III RNase H- Reverse Transcriptase kit (Invitrogen) following the manufacturer's instructions. Reverse transcribed cDNA was stored at $-20^{\circ} \mathrm{C}$, once synthesized.

Microarray construction and probe preparation. The construction of the microarrays used in this study (BioStarH-141s, cDNA microarray) was carried out following the method of Brown. There were 14112 sequences including full-length and partial cDNAs representing known, novel, and control genes provided by United Gene Holdings. All the sequences were verified. The known genes were selected from the NCBI Unigene set and cloned into a plasmid vector. The novel genes were obtained through systematic full-length cloning efforts carried out at United Gene Holding. The control spots of non-human origin in the 14112 chip included the rice U2 RNA gene ( 8 spots), the hepatitis $\mathrm{c}$ virus (HCV) coat protein gene (8 spots), and spotting 
Table I. The information of NPC samples used in this study.

\begin{tabular}{|c|c|c|c|c|c|}
\hline Sample no. & Gender & Age & TNM stage & Clinical cancer stage & Radio-sensitivity \\
\hline \multicolumn{6}{|l|}{ Microarrays } \\
\hline 6 & Male & 34 & $\mathrm{~T} 2 \mathrm{~N} 2 \mathrm{M} 0$ & III & $\mathrm{RS}$ \\
\hline 8 & Male & 65 & T3N1M0 & III & $\mathrm{RS}$ \\
\hline 10 & Male & 51 & T3N0M0 & III & $\mathrm{RS}$ \\
\hline 19 & Male & 60 & $\mathrm{~T} 2 \mathrm{~N} 2 \mathrm{M} 0$ & III & $\mathrm{RS}$ \\
\hline 56 & Male & 51 & $\mathrm{~T} 2 \mathrm{~N} 1 \mathrm{M} 1$ & II & $\mathrm{RS}$ \\
\hline 83 & Male & 54 & T4N1M0 & IV & $\mathrm{RS}$ \\
\hline 102 & Male & 42 & T1N1M0 & II & $\mathrm{RS}$ \\
\hline 130 & Male & 60 & $\mathrm{~T} 2 \mathrm{~N} 2 \mathrm{M} 0$ & III & $\mathrm{RS}$ \\
\hline 22 & Male & 55 & T3N1M0 & III & RR \\
\hline 25 & Male & 41 & T4N2M0 & IV & RR \\
\hline 60 & Male & 45 & $\mathrm{~T} 2 \mathrm{~N} 2 \mathrm{M} 0$ & III & $\mathrm{RR}$ \\
\hline 84 & Male & 51 & $\mathrm{~T} 2 \mathrm{~N} 3 \mathrm{M} 0$ & IV & $\mathrm{RR}$ \\
\hline 87 & Male & 41 & $\mathrm{~T} 3 \mathrm{~N} 2 \mathrm{M} 0$ & III & RR \\
\hline 93 & Male & 38 & T3N0M0 & III & $\mathrm{RR}$ \\
\hline 108 & Female & 28 & T3N2M0 & III & $\mathrm{RR}$ \\
\hline 109 & Female & 50 & T1N1M0 & II & $\mathrm{RR}$ \\
\hline 122 & Male & 67 & T3N3M0 & IV & $\mathrm{RR}$ \\
\hline 157 & Male & 53 & T4N0M0 & IV & $\mathrm{RR}$ \\
\hline 158 & Male & 32 & T1N1M0 & II & $\mathrm{RR}$ \\
\hline 170 & Male & 50 & $\mathrm{~T} 2 \mathrm{~N} 3 \mathrm{M} 0$ & IV & $\mathrm{RR}$ \\
\hline \multicolumn{6}{|c|}{ Quantitative real-time RT-PCR } \\
\hline 90 & Male & 45 & $\mathrm{~T} 2 \mathrm{~N} 2 \mathrm{M} 0$ & III & $\mathrm{RS}$ \\
\hline 155 & Male & 39 & $\mathrm{~T} 3 \mathrm{~N} 2 \mathrm{M} 0$ & III & $\mathrm{RS}$ \\
\hline 178 & Male & 38 & $\mathrm{~T} 3 \mathrm{~N} 2 \mathrm{M} 0$ & III & $\mathrm{RS}$ \\
\hline 204 & Male & 40 & T3N1M0 & III & $\mathrm{RS}$ \\
\hline 206 & Male & 54 & $\mathrm{~T} 2 \mathrm{~N} 1 \mathrm{M} 0$ & II & $\mathrm{RS}$ \\
\hline 209 & Male & 59 & T3N2M0 & III & $\mathrm{RS}$ \\
\hline 215 & Male & 34 & $\mathrm{~T} 4 \mathrm{~N} 1 \mathrm{M} 0$ & IV & $\mathrm{RS}$ \\
\hline 219 & Male & 29 & T3N0M0 & III & $\mathrm{RS}$ \\
\hline 222 & Male & 27 & T2NOM0 & II & $\mathrm{RS}$ \\
\hline 237 & Female & 42 & T4N1M0 & IV & $\mathrm{RS}$ \\
\hline 51 & Male & 33 & T2N0M0 & II & $\mathrm{RR}$ \\
\hline 66 & Male & 49 & T3N1M0 & III & $\mathrm{RR}$ \\
\hline 145 & Male & 52 & T4N0M0 & IV & $\mathrm{RR}$ \\
\hline 185 & Male & 29 & T4N1M0 & IV & $\mathrm{RR}$ \\
\hline 202 & Male & 56 & T4N1M0 & IV & $\mathrm{RR}$ \\
\hline 211 & Female & 43 & T2NOM0 & II & $\mathrm{RR}$ \\
\hline 220 & Male & 40 & T3N3M0 & IV & $\mathrm{RR}$ \\
\hline
\end{tabular}

RS, radiation-sensitive; RR, radiation-resistant.

solution alone without DNA (32 spots). The cDNA inserts were amplified by use of the polymerase chain reaction (PCR) using universal primers to plasmid vector sequences and were then purified. All PCR products were examined by agarose gel electrophoresis to ensure the quality and the identity of the amplified clones as expected. Then the amplified PCR products were dissolved in a buffer containing SSC solution. The solution with amplified PCR products were spotted onto silylated slides (CEL Associates, Houston, TX, USA) using a Cartesian PixSys 7500 motion control 
robot (Cartesian Technologies, Irvine, CA, USA) fitted with ChipMaker Micro-Spotting Technology (TeleChem International, Sunnyvale, CA, USA). The glass slides were then hydrated for $2 \mathrm{~h}$ in $70 \%$ humidity, dried for $0.5 \mathrm{~h}$ at room temperature, and UV cross-linked $(65 \mathrm{mj} / \mathrm{cm})$. They were further processed at room temperature by soaking in $0.2 \%$ sodium dodecyl sulfate (SDS) for $10 \mathrm{~min}$, distilled $\mathrm{H}_{2} \mathrm{O}$ for $10 \mathrm{~min}$, and $0.2 \%$ sodium borohydride $\left(\mathrm{NaBH}_{4}\right)$ for $10 \mathrm{~min}$. The slides were dried again and ready for use. The fluorescent cDNA probes were prepared through reverse transcription of the isolated mRNAs and then purified according to the methods of Schena et al. The RNA samples from nasopharyngeal phlogistic patients were labeled with Cy3-dUTP and those from NPC patients with Cy5-dUTP.

Hybridization. The probe was dissolved in $20 \mu 1$ of hybridization solution [5X SSC (0.75 M NaCl and $0.075 \mathrm{M}$ sodium citrate), $0.4 \%$ SDS, $50 \%$ formamide]. Microarrays were pre-hybridized with a hybridization solution containing $0.5 \mathrm{mg} / \mathrm{ml}$ denatured salmon sperm DNA at $42^{\circ} \mathrm{C}$ for $6 \mathrm{~h}$. Fluorescent probe mixtures were denatured at $95^{\circ} \mathrm{C}$ for $5 \mathrm{~min}$, and then applied onto the pre-hybridized chip under a cover glass. Chips were hybridized at $42^{\circ} \mathrm{C}$ for $15-17 \mathrm{~h}$. Next, the hybridized chips were each washed at $60^{\circ} \mathrm{C}$ for $10 \mathrm{~min}$ in solutions of $2 \mathrm{X} \mathrm{SSC}$ and $0.2 \%$ SDS, 0.1X SSC and $0.2 \%$ SDS, and 0.1X SSC, then dried at room temperature.

Detection and microarray data analysis. The chips were scanned with a ScanArray 4000 (GSI Lumonics, Bellerica, MA) at two wavelengths, 635 and $532 \mathrm{~nm}$, to detect emission from both $\mathrm{Cy} 5$ and $\mathrm{Cy} 3$, respectively. The acquired images were analysed using GenePix Pro 3.0 software. The intensities of each spot at the two wavelengths represent the quantity of Cy3-dUTP and Cy5-dUTP. Ratios of Cy5 to Cy3 were computed using the GenePix Pro 3.0 median of ratio method. Overall intensities were normalized using the corresponding GenePix default normalization factor. All the spots flagged 'bad' or 'not found' by GenePix software were removed from the final data. Only genes with raw intensity values for both $\mathrm{Cy} 3$ and $\mathrm{Cy} 5$ of $>200$ counts were selected from each array and used for further analysis.

Gene expression measures are available at GEO (http:// www.ncbi.nlm.nih.gov/geo/query/acc.cgi?acc=GSE32389). For microarray data analysis, missing values were replaced using the K-nearest neighbours calculation method with $\mathrm{k}=10$, but only when there were $>30 \%$ of values missing per gene.

Firstly, we used two-class unpaired significance analysis of microarray (SAM, version 3.02) to selected genes that were differentially expressed in radiation-resistant NPC patients comparing with radiation-sensitive NPC patients (26). Gene selected following 1000 permutations were those with expression that was $>1.5$-fold different from the mean expression. We also used $\mathrm{q}<0.05$ as an additional discriminating parameter. Then, one-class SAM was used to selected genes that aberrantly differentiated RRNPC and RSNPC patients, respectively, from NPCI. Following 1000 permutations, we select genes using the same discriminating parameters as shown above.

Cluster and TreeView software was used to perform unsupervised hierarchical clustering analysis (27). Pathway analysis was

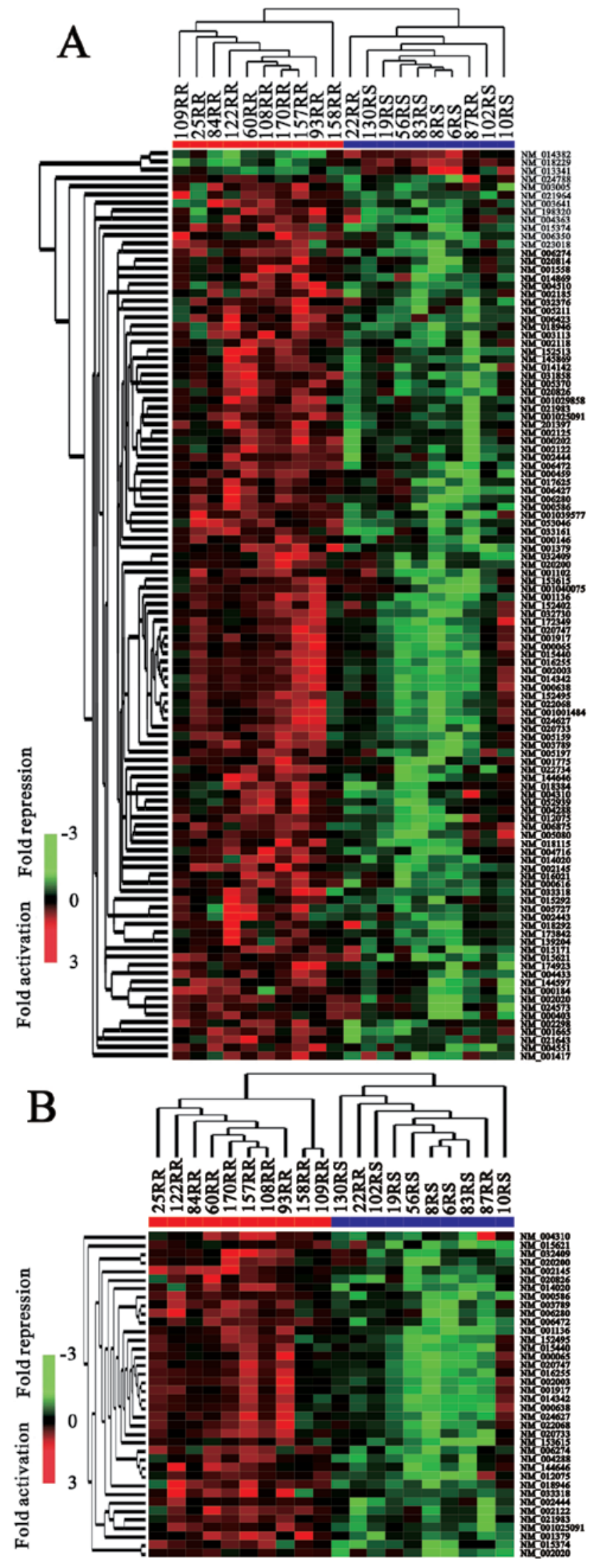

Figure 1. (A) Unsupervised hierarchical clustering of 111 differentially expressed genes, the results of two-class SAM analysis by $\mathrm{q}<0.05$. (B) Unsupervised hierarchical clustering of 38 differentially expressed genes, the results of two-class SAM analysis by $\mathrm{q}<0.01$. Both gene sets are able to cluster the RRNPC patients in one branch, other than patients 22 and 87. Red indicates genes that are over-expressed relative to control, whereas green indicates genes that are under-expressed. Black indicates that gene expression is not changed relative to control. 

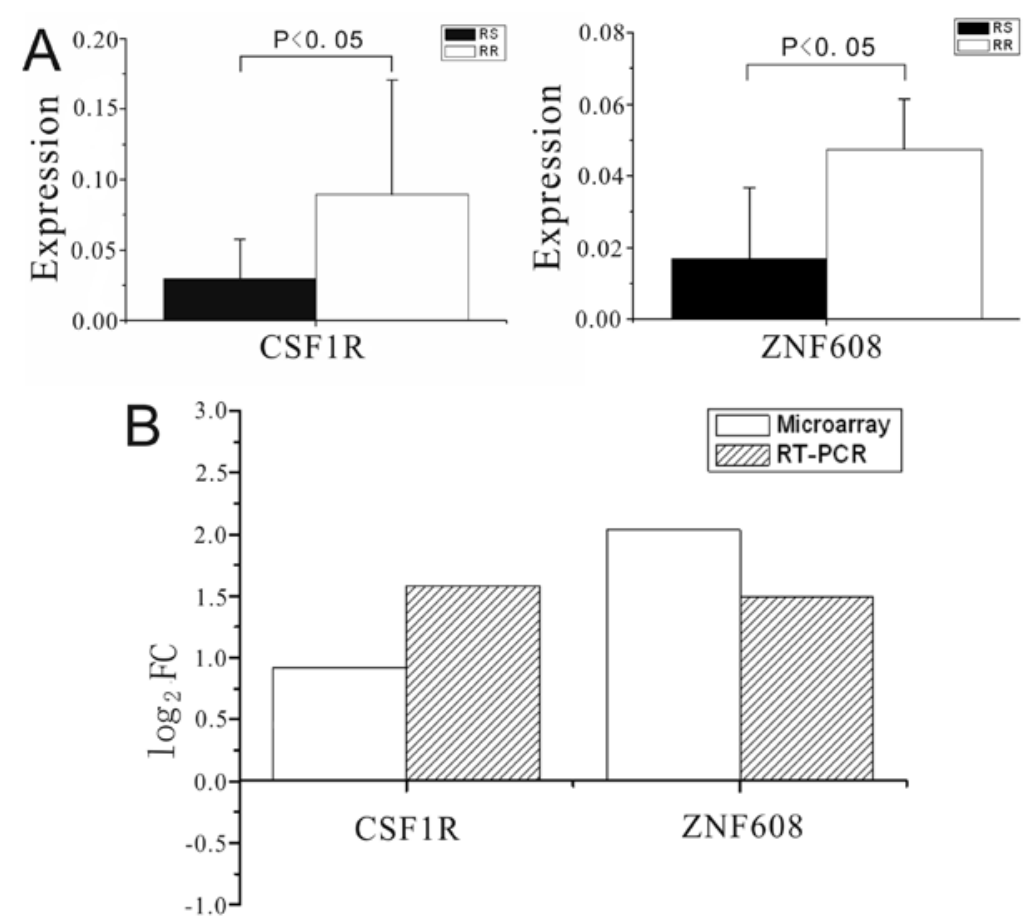

Figure 2. (A) Confirmatory studies of selected genes by real-time RT-PCR. The graph shows that the two aberrant genes (CSF1R and ZNF608) in microarray results also showed significant alteration in real-time RT-PCR. (B) Fold change (FC) comparison between the microarray and real-time RT-PCR in two genes. The $\log _{2}$ FC showed that CSF1R and ZNF608 have the same trends in microarray and real-time RT-PCR.

performed with GenMAPP 2.1 software (Gene Map Annotator and Pathway Profiler). Significantly altered pathways were defined when their $Z$ scores were $>1.96(28,29)$.

Quantitative real-time $R T-P C R$. The quantitative real-time RT-PCR was used to validate our microarray results. Quantitative real-time RT-PCR was performed on ABI 7900HT thermocycler (Applied Biosystems, Foster City, CA, USA) by using 2X HotSybr PCR reaction mix (NuStar, Hong Kong, China). Oligonucleotide primers were: human ZNF608 sense, ccettgttgcctctcagc, and antisense, caatgtacatgtccaatcttgttattc; human CSF1R sense, tctggtcctatggcatcctc, and antisense, gatgccagggtagggattc; human GAPDH sense, agccacatcgctcagacac, and antisense, gcccaatacgaccaaatcc. After $10 \mathrm{~min}$ of initial denaturation at $95^{\circ} \mathrm{C}$, the cycling protocol was 40 cycles of denaturation at $95^{\circ} \mathrm{C}$ $(15 \mathrm{sec})$, annealing and elongation at $60^{\circ} \mathrm{C}(1 \mathrm{~min})$. On each run, we quantified all samples according to the Sequence Detection System (SDS) software program, version 2.3 (Applied Biosystems). The levels of mRNA for ZNF608 and CSF1R were corrected with GAPDH housekeeping control amplification. The $2^{-\mathrm{ACt}}$ method was used as relative quantification measure of differential expression. Means and standard deviations of individual groups $(n=3)$ were calculated. P-values were assessed by performing two-sided Wilcoxon rank sum test with the SPSS software (version 13).

\section{Results}

Differentially expressed genes between RRNPC and RSNPC patients. After normalization, we selected 9646 genes with signals present on at least 14 microarray data for SAM analysis to identify genes with expression profiles that differentiate radiation-resistant NPC (RRNPC) patients from radiationsensitive NPC (RSNPC) patients. Preliminary two-class SAM was preformed, comparing RRNPC patient data to RSNPC patient data. Interestingly, we found that two RRNPC patients, no. 22 and 87, were clustered within RSNPC branch and were not grouped to RRNPC branch. All the patients were reviewed by otorhinolaryngology pathologist of the People's Hospital of Guangxi Zhuang Nationality Autonomous Region, according to clinical radiation sensitivity classification. To avoid heterogeneity bias associated with particular patients and to highlight general processes, patients no. 22 and 87 were withdrawn from further analysis.

Therefore, 10 RRNPC and 8 RSNPC were used to select differentially expressed genes. To increase the robustness of selected genes, two cut-off criteria were applied simultaneously, consistent with a 1.5 -fold change ( $\mathrm{FC}>1.5)$ and with $\mathrm{q}<0.05$. This gave a set of 111 genes, including 108 over-expressed genes and 3 under-expressed genes in RRNPC patients (Fig. 1A). Of them, 38 genes were $\mathrm{q}<0.01$ and all of them were over-expressed in RRNPC patients (Fig. 1B). This gene set was used for hierarchical clustering representation of patients. Both of them were able to cluster the RRNPC patients except patients no. 22 and 87 , in one branch.

Validation of microarray results using the other independent samples by quantitative real-time RT-PCR.ZNF608 and CSF1R were found over-expressed in RRNPC patients compared with RSNPC patients, and the fold change (FC) was 4.10-and 1.89fold, respectively.

These two genes were chosen for validation using real-time RT-PCR with the other independent 10 RSNPC and 7 RRNPC patient specimens. ZNF608 and CSF1R were validated as 
Table II. Statistically enriched pathways by targets of differentially expressed genes in RRNPC.

\begin{tabular}{|c|c|c|c|c|c|}
\hline Function category & Enriched pathway name & $\begin{array}{l}\text { Number } \\
\text { changed }\end{array}$ & $\begin{array}{l}\text { Number } \\
\text { measured }\end{array}$ & $\begin{array}{l}\text { Percent } \\
\text { changed }\end{array}$ & Z score \\
\hline \multirow{3}{*}{$\begin{array}{l}\text { Cytokines and } \\
\text { inflammatory }\end{array}$} & Cytokine activity ${ }^{\mathrm{a}}$ & 4 & 40 & 10 & 4.113 \\
\hline & $\begin{array}{l}\text { Hs_Cytokines_and_Inflammatory_Response_ } \\
\text { (BioCarta)_WP530_35247 a }\end{array}$ & 3 & 8 & 37.5 & 7.896 \\
\hline & Hs_Inflammatory_Response_Pathway ${ }^{\mathrm{a}}$ & 2 & 13 & 15.38 & 3.853 \\
\hline \multirow[t]{2}{*}{ Humoral immune } & Humoral immune response ${ }^{\mathrm{a}}$ & 4 & 43 & 9.30 & 3.908 \\
\hline & Humoral defense mechanism (sensu Vertebrata) ${ }^{a}$ & 4 & 31 & 12.90 & 4.88 \\
\hline \multirow[t]{4}{*}{ Cell ion homeostasis } & Cell homeostasis & 5 & 24 & 20.83 & 7.323 \\
\hline & Cell ion homeostasis & 4 & 21 & 19.04 & 6.21 \\
\hline & Ion homeostasis & 4 & 23 & 17.39 & 5.88 \\
\hline & di-, tri-valent inorganic cation transport & 2 & 29 & 6.89 & 2.194 \\
\hline \multirow[t]{2}{*}{ Membrane system } & Endomembrane system ${ }^{\mathrm{a}}$ & 6 & 98 & 6.12 & 3.456 \\
\hline & Plasma membrane & 6 & 79 & 7.59 & 4.124 \\
\hline \multirow[t]{3}{*}{ Cell proliferation } & Regulation of cell proliferation & 4 & 87 & 4.59 & 2.137 \\
\hline & Positive regulation of cell proliferation & 3 & 37 & 8.10 & 3.056 \\
\hline & Growth factor activity & 2 & 29 & 6.89 & 2.194 \\
\hline \multirow[t]{3}{*}{ Receptor protein signaling } & Receptor signaling protein activity & 3 & 61 & 4.91 & 1.982 \\
\hline & Enzyme linked receptor protein signaling pathway & 3 & 60 & 5 & 2.015 \\
\hline & $\begin{array}{l}\text { Transmembrane receptor protein tyrosine kinase } \\
\text { signaling pathway }\end{array}$ & 3 & 50 & 6 & 2.391 \\
\hline \multirow[t]{2}{*}{ One carbon metabolism } & Hs_Folate_WP176_36214 ${ }^{\mathrm{a}}$ & 3 & 31 & 9.67 & 3.478 \\
\hline & Hs_One_Carbon_Metabolism_WP241_36181 ${ }^{\mathrm{a}}$ & 2 & 17 & 11.76 & 3.244 \\
\hline \multirow[t]{2}{*}{ Carbohydrate binding } & Sugar binding & 3 & 38 & 7.89 & 2.995 \\
\hline & Carbohydrate binding $^{\mathrm{a}}$ & 4 & 63 & 6.34 & 2.904 \\
\hline \multirow[t]{5}{*}{ Other } & Extracellular space ${ }^{a}$ & 6 & 91 & 6.59 & 3.681 \\
\hline & Structural constituent of cytoskeleton & 3 & 46 & 6.52 & 2.569 \\
\hline & Hs_GPCRDB_Class_A_Rhodopsin-like & 2 & 28 & 7.14 & 2.258 \\
\hline & I-kappaB kinase NF-kappaB cascade & 3 & 56 & 5.35 & 2.155 \\
\hline & taxis & 2 & 33 & 6.06 & 1.967 \\
\hline
\end{tabular}

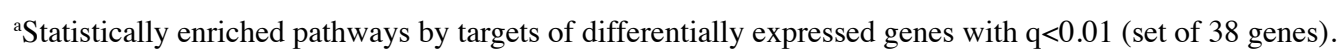

up-regulation in RRNPC compared with RSNPC patients (Fig. 2A). The fold changes of each gene from real-time RT-PCR and microarray are shown in Fig. 2B. Taken together, it is indicated that real-time RT-PCR results are consistent with our microarray data, and these two genes may act as markers for prediction of the radiation sensitivity of NPC before radiation treatment.
Identification of genes characterizing pathways specific to RRNPC compared with RSNPC. To further study the biological processes, we investigated the molecular pathways depicting RRNPC by characterizing differentially expressed genes identified above. Nine categories, containing 26 biological pathways, were revealed $(Z$ score $>1.96$ ): cytokines and inflammatory response, humoral immune, cell ion homeostasis, membrane system, cell proliferation, receptor protein signalling pathway, 


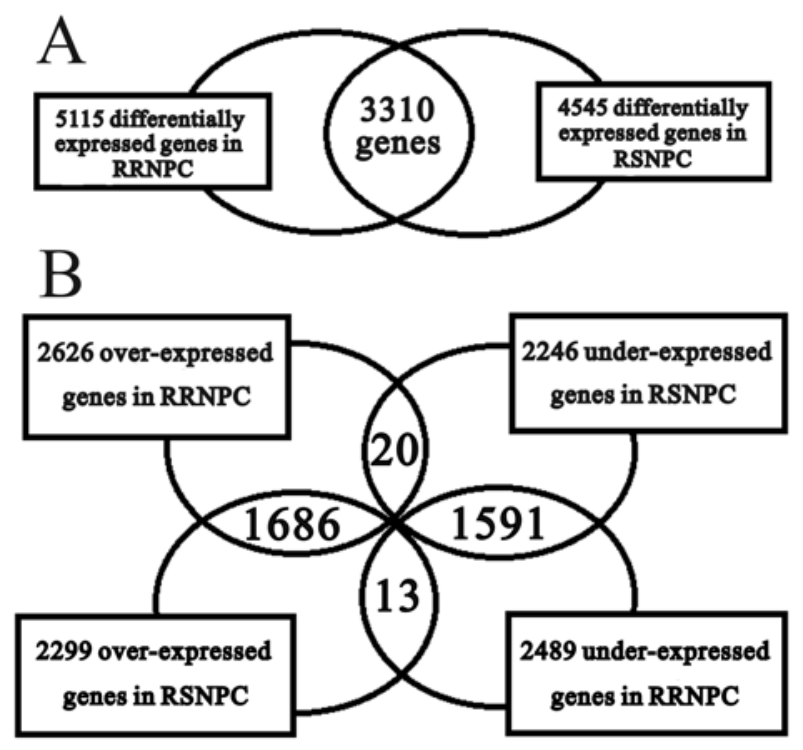

Figure 3. Venn diagram of the number of differentially expressed genes in two patients groups. (A) Schematic representation of selected genes differentially expressed in RRNPC or RSNPC compared with NPCI. Compared with NPCI, 5115 genes and 4545 genes $(\mathrm{q}<0.05)$ were aberrantly expressed in RRNPC and RSNPC, respectively. Of them, 3310 genes were differentially expressed in both. (B) Compared with NPCI, 1686 genes congruous up-regulation and 1591 genes congruous down-regulation in both. However, 33 genes showed contrary expression trend in two kinds of NPC groups, 20 genes over-expressed in RRNPC, however, under-expressed in RSNPC, and 13 genes under-expressed in RRNPC whereas over-expressed in RRNPC.

one carbon metabolism, carbohydrate binding, and others (Table II). Ten enriched biological pathways were obtained from the 38 gene $(\mathrm{q}<0.01)$ and 111 gene $(\mathrm{q}<0.05)$ sets, whereas the other biological processes concerned only the set of 111 genes $(\mathrm{q}<0.05)$.

Identification of genes ectopically expressed in RRNPC and $R S N P C$ compared with NPCI, respectively. To further identify genes with expression profile that differentiate RRNPC or RSNPC patients from NPCI, we selected 9646 genes with signals present on at least 14 microarray data for one-class SAM analysis.

Ten RRNPC patient data, other than patients no. 22 and 87, were used to compare with NPCI. Genes (5115) $(\mathrm{q}<0.05$, set-A) were found differentially expressed in RRNPC, including 2626 up-regulated genes and 2489 down-regulated genes. Of them, 3489 genes were $\mathrm{q}<0.01,1810$ genes were over-expressed and 1679 genes were under-expressed.

Moreover, 8 RSNPC patient data were used to compare with NPCI. 4545 genes $(\mathrm{q}<0.05$, set-B) were found aberrantly expressed in RSNPC patients, including 2299 up-regulated genes and 2246 down-regulated genes. Of them, 3252 genes were $\mathrm{q}<0.01,1617$ genes were over-expressed and 1635 were under-expressed.

Identification of genes aberrantly expressed in both sets of NPC patients compared with NPCI. To further explore genes aberrantly expressed in both sets of NPC patients, we compared two gene sets, set-A and set-B. Here, 3310 genes $(q<0.05)$ were identified differentially expressed in both sets of NPC patients compared with NPCI (Fig. 3). Of them, 3277 genes show

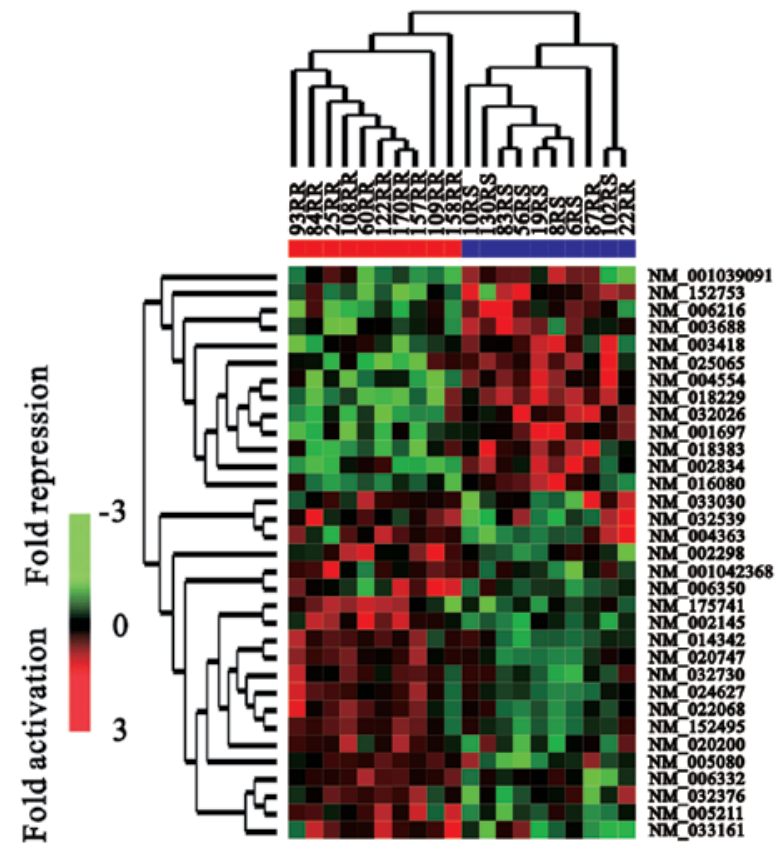

Figure 4. Unsupervised hierarchical clustering of 33 differentially expressed genes have different expression trend in 8 RSNPC and 12 RRNPC compared NPCI. This set of genes is able to cluster the RRNPC patients in one branch, other than the patients 22 and 87. Red, genes that are over-expressed relative to control; green, genes that are under-expressed. Black, gene expression unchanged relative to control.

coherent expression pattern in two patients groups, including 1686 up-regulated genes and 1591 down-regulated genes. Interestingly, 33 genes showed contrary expression trend, of which 20 genes $(\mathrm{q}<0.05)$ were found over-expressed in RRNPC whereas under-expressed in RSNPC, and 13 genes were found under-expressed in RRNPC, however, over-expressed in RSNPC (Fig. 3B). This set of 33 genes was able to cluster the RRNPC patients in one branch, other than patients no. 22 and 87 (Fig. 4). Among them, 3 differentially expressed genes (ZNF608, PIZEO2 and CSF1R) were identified over-expressed in RRNPC patients $(\mathrm{q}<0.01)$, however under-expressed in RSNPC patients $(\mathrm{q}<0.01)$, compared with NPCI.

Identification of genes only aberrantly expressed in RRNPC and RSNPC patients compared with NPCI, respectively. Compared with NPCI, 1805 genes $(q<0.05,35.29 \%)$ were only differentially expressed in RRNPC, including 921 up-regulated genes and 884 down-regulated genes. In contrast, 1235 genes $(q<0.05,27.17 \%)$ were only aberrantly expressed in RSNPC, including 600 up-regulated and 635 down-regulated genes.

Taken together with our previous data, a set of 1838 genes ( $<<0.05,35.93 \%$, set-C) was identified specifically aberrantly expressed in RRNPC, including 1805 genes which only differentially expressed in RRNPC and 33 genes which have contrary expression trend between RRNPC and RSNPC compared with NPCI. Of them, 941 genes $(\mathrm{q}<0.05,18.40 \%)$ were up-regulated and 897 genes $(\mathrm{q}<0.05,17.54 \%)$ were down-regulated only in RRNPC compared with NPCI.

Moreover, a set of 1268 RSNPC specific genes $(\mathrm{q}<0.05$, $27.90 \%$, set-D) was identified, including 1235 genes which only differentially expressed in RSNPC and 33 contrary expression trend genes. Of them, 613 genes $(\mathrm{q}<0.05,13.49 \%)$ were up-regu- 
Table III. Statistically enriched pathways by targets of differentially expressed gene set-C and set-D, respectively.

\begin{tabular}{|c|c|c|c|c|}
\hline $\begin{array}{l}\text { A, Statistically enriched pathways by targets o } \\
\text { Enriched pathway name }\end{array}$ & $\begin{array}{l}\text { sed gene } \\
\text { Number } \\
\text { changed }\end{array}$ & $\begin{array}{l}\text { Number } \\
\text { measured }\end{array}$ & $\begin{array}{l}\text { Percent } \\
\text { changed }\end{array}$ & $\mathrm{Z}$ score \\
\hline Hs_Ribosomal_Proteins ${ }^{a}$ & 48 & 61 & 78.68 & 4.254 \\
\hline Enzyme inhibitor activity & 50 & 77 & 64.93 & 2.355 \\
\hline Regulation of protein kinase activity & 32 & 47 & 68.08 & 2.269 \\
\hline Protein amino acid dephosphorylation & 34 & 52 & 65.38 & 1.996 \\
\hline Dephosphorylation & 36 & 54 & 66.66 & 2.224 \\
\hline Hs_Phosphatidylinositol_signaling_system & 39 & 59 & 66.10 & 2.239 \\
\hline Inorganic anion transport & 22 & 31 & 70.96 & 2.162 \\
\hline Anion transport & 29 & 42 & 69.04 & 2.269 \\
\hline Cytosol $^{\mathrm{a}}$ & 91 & 150 & 60.66 & 2.248 \\
\hline Hs_Smooth_muscle_contraction & 46 & 71 & 64.78 & 2.236 \\
\hline Hs_Glucuronidation_WP698_33413 ${ }^{\mathrm{a}}$ & 10 & 12 & 83.33 & 2.201 \\
\hline Cell-cell adhesion & 26 & 38 & 68.42 & 2.08 \\
\hline Cell junction & 18 & 25 & 72 & 2.044 \\
\hline Hs_IL-5_Signaling_Pathway_WP127_35644 & 27 & 40 & 67.5 & 2.018 \\
\hline
\end{tabular}

B, Statistically enriched pathways by targets of differentially expressed gene set-C.

\begin{tabular}{|c|c|c|c|c|}
\hline Enriched pathway name & $\begin{array}{l}\text { Number } \\
\text { changed }\end{array}$ & $\begin{array}{l}\text { Number } \\
\text { measured }\end{array}$ & $\begin{array}{l}\text { Percent } \\
\text { changed }\end{array}$ & Z score \\
\hline Hs_Ribosomal_Proteins ${ }^{\mathrm{a}}$ & 55 & 61 & 90.16 & 5.23 \\
\hline Cytosol $^{\mathrm{a}}$ & 104 & 150 & 69.33 & 3.042 \\
\hline Hs_Glucuronidation_WP698_33413 ${ }^{\mathrm{a}}$ & 12 & 12 & 100 & 2.999 \\
\hline Hs_p38_MAPK_signaling_pathway & 17 & 19 & 89.47 & 2.848 \\
\hline Transferase activity, transferring hexosyl groups & 32 & 42 & 76.19 & 2.496 \\
\hline Hs_IL-4_signaling_Pathway_WP395_35643 & 24 & 31 & 77.41 & 2.281 \\
\hline Hs_TGF-beta_Receptor_Signaling_Pathway_WP366_35302 & 62 & 91 & 68.13 & 2.124 \\
\hline Hs_Glycine_serine_and_threonine_metabolism & 14 & 17 & 82.35 & 2.099 \\
\hline Hs_Estrogen_metabolism_WP697_33412 & 9 & 10 & 90 & 2.098 \\
\hline Apoptosis & 76 & 114 & 66.66 & 2.063 \\
\hline Circulation & 20 & 26 & 76.92 & 2.037 \\
\hline Hs_Calcium_regulation_in_cardiac_cells & 42 & 60 & 70 & 2.014 \\
\hline Extracellular matrix (sensu Metazoa) & 29 & 40 & 72.5 & 1.962 \\
\hline
\end{tabular}

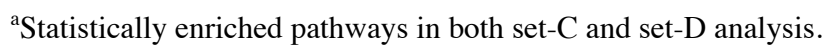

lated and 655 genes $(\mathrm{q}<0.05,14.41 \%)$ were down-regulated only in RSNPC comparing with NPCI. These two sets of genes were recruited for further pathway analysis.

Identification of genes characterizing pathways specific to $R R N P C$ and RSNPC compared with NPCI, respectively. Set-C and set-D genes were used to investigate the biological pathways specific to RRNPC and RSNPC, respectively. Filtering by $\mathrm{Z}$ score $>1.96,13$ and 14 enriched biological pathways were revealed, respectively (Table III).

Interestingly, 3 common biological processes appeared in both RRNPC and RSNPC patients compared with NPCI: ribosomal protein, cytosol, and glucuronidation. Thus, 10 biological pathways were specifically enriched in RRNPC, and 11 biological pathways were specifically found in RSNPC (Table III).

\section{Discussion}

Nasopharyngeal carcinoma is a special type of squamous cell carcinoma of head and neck associated with EBV infection, environmental factors and genetic aberrance (3-5). Radiotherapy is the major treatment modality for NPC, but in some cases, the disease is radiation-resistant, indicating radiation resistance remains a serious obstacle to successful treatment in many cases. Currently, more attention is focused on the study of nasopharyngeal carcinoma cell lines (20-24). However, the knowledge on 
the difference between RSNPC and RRNPC tissues remains limited.

In the present study, we firstly focused on aberrance of gene expression in RRNPC compared with RSNPC patient tissue by DNA microarray. Class comparison analysis revealed 111 differentially expressed genes $(\mathrm{q}<0.05)$, with at least expression in 14 specimens, with 1.5 -fold change, of which 38 with a significance of $\mathrm{q}<0.01$. However, previously no overlap was found to be involved in radiation resistance in NPC. This may be because of distinct specificity between cells and tissues, but it is also possible that there exist fundamental mechanisms underlying radiation resistance that have not yet been elucidated.

In these aberrant expression genes, ZNF608 and CSF1R, were validated by quantitative real-time RT-PCR in 17 independent NPC patient specimens, indicating they may act as markers for prognosis of radiation sensitivity of NPC. Colony stimulating factor 1 receptor (CSF1R), which contains a split kinase domain and number of intracellular tyrosine residues that are phosphorylated upon activation of the receptor (30), encoded by the $c f m s$ proto-oncogene. It interacts with colony stimulating factor-1 (CSF-1) regulating the proliferation and differentiation of cells of the mononuclear phagocytic lineage (31). In addition to its normal role in mononuclear phagocyte biology, CSF-1 and CSF1R are involved in the tumourigenicity of epithelial cells as their elevated expression has been found in breast, uterine, and ovarian tumour cells, and the extent of expression in these tumours correlates high grade and poor prognosis (32-34). Most notably, CSF-1/CSF1R has been shown to promote tumour invasion and metastasis through interaction of tumour-associated macrophages and carcinoma cells mediated by a CSF-1-EGF paracrine loop (35-38). Nevertheless, there is no study on CSF1R aberrant expression in NPC or between RSNPC and RRNPC until now. In our data, over-expression of CSF1R in RR-NPC was found, indicating the capacity of metastasis and invasion in RRNPC was more than RSNPC in patient tissues. Zinc finger protein 608 (ZNF608) is a member of zinc finger protein family. Its detailed function remains unclear, however, over-expression of this gene in RRNPC indicates it may act as a marker for prognosis of radiotherapy. Interestingly, these two genes were also over-expressed in RRNPC patients, but under-expressed in RSNPC patients compared to NPCI. Due to lack enough NPCI patient specimens, we could not further validate the differential expression of these genes in NPCI patients.

Pathway analysis enables us to study the biological processes enriched by differential expression genes. We propose 3 mostly biological processes may act as potential mechanisms underlying RRNPC patients. Firstly, we noted that cell ion homeostasis, especially di-, tri-valent inorganic cation transport, changed in RRNPC patients. The report of Heise et al (39), suggested that ionizing radiation-stimulated cation channel activation, $\mathrm{Ca}^{2+}$ entry and calmodulin-dependent kinase II activity participate in control of cell cycle progression and survival of irradiated chronic myeloid leukaemia cells. It is well known that cell cycle progression of proliferating cells requires $\mathrm{Ca}^{2+}$ signals to enter and accomplish the $\mathrm{S}$ and the $\mathrm{M}$ phase of the cell cycle $(40,41)$. Nevertheless, $\mathrm{Ca}^{2+}$ signals may also trigger programmed cell death. Tumour cells have been postulated to resolve this dilemma by remodelling their $\mathrm{Ca}^{2+}$ signalosome. In this case, possibly hazardous $\mathrm{Ca}^{2+}$ signals generated by bulkly release of $\mathrm{Ca}^{2+}$ from the endoplasmic reticulum are replaced by signals arising from slower $\mathrm{Ca}^{2+}$ entry and extrusion across the plasma membrane. These plasma membrane-born signals are generated by store independent $\mathrm{Ca}^{2+}$ channels and the activity of $\mathrm{Ca}^{2+}$ pumps or anti-porters, respectively (42). Interestingly, PIZEO2, encoding a component of cation channels localized on plasma membrane (43), is differentially expressed in our data, and may act as a novel $\mathrm{Ca}^{2+}$ channel to rescue tumour cells and promote cell proliferation under radiation therapy. This may lead to the changes of membrane system in RRNPC patients.

Secondly, humoral immunity was also significantly altered in RRNPC patients. We suggest that this is probably due to CSF1R interacting with tumour-associated macrophages, and then inducing the change of cytokines as well as inflammatory response. Recently, Lenzo et al (44) found CSF1R could as a marker for the development of macrophage-lineage cells. Together with our data, we suggest that the radiation sensitivity of NPC patients may relate to the development of macrophagelineage cells. In addition, ectopic expression of CSF1R could promote tumour invasion and metastasis (35-38), which may lead to the alteration of extracellular space and structural constituent of cytoskeleton.

Thirdly, trans-membrane receptor protein tyrosine kinase signalling pathway was also varied in RRNPC patients comparing with RSNPC. Receptor protein tyrosine kinase signalling pathway is widely reported differentially expressed in various cancers, and its over-expression promoted cell transformation, proliferation, oncogenesis, tumour development, and anti-apoptosis activity (45). We suggest that this pathway mostly promote RRNPC cell proliferation as well as anti-apoptosis activity under radiotherapy.

Additionally, some metabolism also changed, such as one carbon metabolism and carbohydrate binding. Together with our microarray data, we suggest the radiation-resistant capacity of NPC was mostly due to the change of cell $\mathrm{Ca}^{2+}$ homeostasis, promoting anti-apoptosis, DNA repair and rescuing tumour cells under radiation therapy. Cell proliferation promotion induced by extracellular and intracellular factors may maintain tumour size under radiotherapy, and leads to recurrence after treatment.

We explored the difference between two kinds of NPC and NPCI. Comparing with NPCI, RRNPC patients have greater expression difference than RSNPC patients. To characterize which biological processes were changed, genes which in set-C and set-D were used to analyse biological pathways, respectively. Interestingly, 3 biological pathways changed in both sets of NPC patients, ribosomal proteins, cytosol and glucuronidation, indicating different ectopically expressed genes could cause the same biological pathway change by interference with various biological effects. We focus on the biological pathways which only changed in RRNPC. Notably, calcium regulation was identified in RRNPC patients, despite the variation of anion transport in RSNPC patients. These data further demonstrated our hypothesis of intracellular $\mathrm{Ca}^{2+}$ homeostasis, which may be a probably process causing the resistance of radiotherapy. We noted that p38 mitogen-activated protein kinase (MAPK) signalling pathway was specific in RRNPC only, indicating some cytokines and inflammatory response were activated $(46,47)$. Mounting evidence suggests that p38 MAPK may have oncogenic functions that are mediated by its involvement in key processes of cancer progression, such as proliferation, invasion, 
inflammation and angiogenesis (48). Additionally, p38 MAPK has been implicated in the $\mathrm{G}_{2} / \mathrm{M}$ checkpoint, which induces cell cycle arrest and facilitates DNA repair. This function may antagonize radiotherapy-induced DNA damage, which could also lead to apoptosis resistance in cancer cells (49). Besides, p38 MAPK has been shown to induce apoptosis in some cells, but plays an anti-apoptosis role in a number of other cell type $(50,51)$. In RSNPC patients, protein dephosphorylation is particularly evident, indicating some pathways, which triggered by dephosphorylation, were activated.

In conclusion, we found 111 genes as markers for prognosis of the radiation sensitivity of NPC patients, CSF1R and ZNF608 were evaluated by real-time RT-PCR using more samples, and we propose 3 probable biological processes, which could cause radiation resistance. We expect our analysis to provide information to further study the mechanism of NPC radio-resistance. We plan to explore whether these biological processes exert radiation-resistant effects, and their detailed mechanism.

\section{Acknowledgements}

This research was supported by grant 30860081 from the National Natural Science Funds and the Guangxi Science and Technology Key Project (no. 0719006-2-4). This research was also supported by the Guangxi Natural Science Funds (no. 0832208) and by the Medical Health Key Research Project of Guangxi (no. 200808).

\section{References}

1. Wei WI and Sham JS: Nasopharyngeal carcinoma. Lancet 365 : 2041-2054, 2005.

2. Hildesheim A and Levine PH: Etiology of nasopharyngeal carcinoma: a review. Epidemiol Rev 15: 466-485, 1993.

3. Tao Q and Chan AT: Nasopharyngeal carcinoma: molecular pathogenesis and therapeutic developments. Expert Rev Mol Med 9: 1-24, 2007.

4. Zheng X, Yan L, Nilsson B, Eklund G and Drettner B: EpsteinBarr virus infection, salted fish and nasopharyngeal carcinoma. A case-control study in southern China. Acta Oncol 33: $867-872,1994$

5. zur Hausen H, Schulte-Holthausen H, Klein G, et al: EBV DNA in biopsies of Burkitt tumours and anaplastic carcinomas of the nasopharynx. Nature 228: 1056-1058, 1970.

6. Yu MC and Yuan JM: Epidemiology of nasopharyngeal carcinoma. Semin Cancer Biol 12: 421-429, 2002.

7. Nielsen NH, Mikkelsen F and Hansen JP: Nasopharyngeal cancer in Greenland. The incidence in an Arctic Eskimo population. Acta Pathol Microbiol Scand A 85: 850-858, 1977.

8. Dickson RI and Flores AD: Nasopharyngeal carcinoma: an evaluation of 134 patients treated between 1971-1980. Laryngoscope 95: 276-283, 1985.

9. Yeh SA, Tang Y, Lui CC, Huang YJ and Huang EY: Treatment outcomes and late complications of 849 patients with nasopharyngeal carcinoma treated with radiotherapy alone. Int J Radiat Oncol Biol Phys 62: 672-679, 2005.

10. DeNittis AS, Liu L, Rosenthal DI and Machtay M: Nasopharyngeal carcinoma treated with external radiotherapy, brachytherapy, and concurrent/adjuvant chemotherapy. Am J Clin Oncol 25: 93-95, 2002.

11. Lee AW, Poon YF, Foo W, et al: Retrospective analysis of 5037 patients with nasopharyngeal carcinoma treated during 19761985: overall survival and patterns of failure. Int J Radiat Oncol Biol Phys 23: 261-270, 1992.

12. Leung SF, Teo PM, Shiu WW, Tsao SY and Leung TW: Clinical features and management of distant metastases of nasopharyngeal carcinoma. J Otolaryngol 20: 27-29, 1991.

13. Li T, Chen JX, Fu XP, et al: microRNA expression profiling of nasopharyngeal carcinoma. Oncol Rep 25: 1353-1363, 2011.
14. Xiong S, Wang Q, Zheng L, Gao F and Li J: Identification of candidate molecular markers of nasopharyngeal Carcinoma by tissue microarray and in situ hybridization. Med Oncol: November 2010 (E-pub ahead of print).

15. Wong YF, Sahota DS, Cheung TH, et al: Gene expression pattern associated with radiotherapy sensitivity in cervical cancer. Cancer J 12: 189-193, 2006.

16. Ogawa K, Utsunomiya T, Mimori K, et al: Differential gene expression profiles of radioresistant pancreatic cancer cell lines established by fractionated irradiation. Int J Oncol 28: 705-713, 2006.

17. Higo M, Uzawa K, Kouzu Y, et al: Identification of candidate radioresistant genes in human squamous cell carcinoma cells through gene expression analysis using DNA microarrays. Oncol Rep 14: 1293-1298, 2005.

18. Guo WF, Lin RX, Huang $\mathrm{J}$, et al: Identification of differentially expressed genes contributing to radioresistance in lung cancer cells using microarray analysis. Radiat Res 164: 27-35, 2005.

19. Fukuda K, Sakakura C, Miyagawa K, et al: Differential gene expression profiles of radioresistant oesophageal cancer cell lines established by continuous fractionated irradiation. Br J Cancer 91: 1543-1550, 2004.

20. Chang JT, Chan SH, Lin CY, et al: Differentially expressed genes in radioresistant nasopharyngeal cancer cells: gp96 and GDF15. Mol Cancer Ther 6: 2271-2279, 2007.

21. Guo Y, Zhu XD, Qu S, et al: Identification of genes involved in radioresistance of nasopharyngeal carcinoma by integrating gene ontology and protein-protein interaction networks. Int J Oncol 40: 85-92, 2011.

22. Feng XP, Yi H, Li MY, et al: Identification of biomarkers for predicting nasopharyngeal carcinoma response to radiotherapy by proteomics. Cancer Res 70: 3450-3462, 2010.

23. Qu Y, Zhang $\mathrm{H}$, Zhao S, Hong J and Tang C: The effect on radioresistance of manganese superoxide dismutase in nasopharyngeal carcinoma. Oncol Rep 23: 1005-1011, 2010.

24. He YX, Zhong PP, Yan SS, et al: DNA-dependent protein kinase activity and radiosensitivity of nasopharyngeal carcinoma cell lines CNE1/CNE2. Sheng Li Xue Bao 59: 524-533, 2007.

25. Schwarz SB, Schaffer PM, Kulka U, Ertl-Wagner B, Hell R and Schaffer M: The effect of radio-adaptive doses on HT29 and GM637 cells. Radiat Oncol 3: 12, 2008.

26. Tusher VG, Tibshirani R and Chu G: Significance analysis of microarrays applied to the ionizing radiation response. Proc Natl Acad Sci USA 98: 5116-5121, 2001.

27. Eisen MB, Spellman PT, Brown PO and Botstein D: Cluster analysis and display of genome-wide expression patterns. Proc Natl Acad Sci USA 95: 14863-14868, 1998.

28. Dahlquist KD, Salomonis N, Vranizan K, Lawlor SC and Conklin BR: GenMAPP, a new tool for viewing and analyzing microarray data on biological pathways. Nat Genet 31: 19-20, 2002.

29. Doniger SW, Salomonis N, Dahlquist KD, Vranizan K, Lawlor SC and Conklin BR: MAPPFinder: using Gene Ontology and GenMAPP to create a global gene-expression profile from microarray data. Genome Biol 4: R7, 2003.

30. Yu W, Chen J, Xiong Y, et al: CSF-1 receptor structure/function in MacCsf11 $\mathrm{r}^{-1-}$ macrophages: regulation of proliferation, differentiation, and morphology. J Leukoc Biol 84: 852-863, 2008.

31. Pixley FJ and Stanley ER: CSF-1 regulation of the wandering macrophage: complexity in action. Trends Cell Biol 14: 628-638, 2004.

32. Kacinski BM: CSF-1 and its receptor in ovarian, endometrial and breast cancer. Ann Med 27: 79-85, 1995.

33. Smith HO, Anderson PS, Kuo DY, et al: The role of colonystimulating factor 1 and its receptor in the etiopathogenesis of endometrial adenocarcinoma. Clin Cancer Res 1: 313-325, 1995.

34. Lin EY, Nguyen AV, Russell RG and Pollard JW: Colonystimulating factor 1 promotes progression of mammary tumors to malignancy. J Exp Med 193: 727-740, 2001.

35. Wyckoff J, Wang W, Lin EY, et al: A paracrine loop between tumor cells and macrophages is required for tumor cell migration in mammary tumors. Cancer Res 64: 7022-7029, 2004.

36. Goswami S, Sahai E, Wyckoff JB, et al: Macrophages promote the invasion of breast carcinoma cells via a colony-stimulating factor-1/epidermal growth factor paracrine loop. Cancer Res 65: 5278-5283, 2005.

37. Condeelis J and Pollard JW: Macrophages: obligate partners for tumor cell migration, invasion, and metastasis. Cell 124: 263-266, 2006 
38. Sampaio NG, Yu W, Cox D, et al: Phosphorylation of CSF-1R Y721 mediates its association with PI3K to regulate macrophage motility and enhancement of tumor cell invasion. J Cell Sci 124: 2021-2031, 2011.

39. Heise N, Palme D, Misovic M, et al: Non-selective cation channel-mediated $\mathrm{Ca}^{2+}$-entry and activation of $\mathrm{Ca}^{2+} /$ calmodulindependent kinase II contribute to G2/M cell cycle arrest and survival of irradiated leukemia cells. Cellular physiology and biochemistry. Int J Exp Cell Physiol Biochem Pharmacol 26: 597-608, 2010

40. Steinhardt RA and Alderton J: Intracellular free calcium rise triggers nuclear envelope breakdown in the sea urchin embryo. Nature 332: 364-366, 1988

41. Taylor JT, Zeng XB, Pottle JE, et al: Calcium signaling and T-type calcium channels in cancer cell cycling. World J Gastroenterol 14: 4984-4991, 2008.

42. Roderick HL and Cook SJ: $\mathrm{Ca}^{2+}$ signalling checkpoints in cancer: remodelling $\mathrm{Ca}^{2+}$ for cancer cell proliferation and survival. Nat Rev Cancer 8: 361-375, 2008.

43. Coste B, Mathur J, Schmidt M, et al: Piezo1 and Piezo2 are essential components of distinct mechanically activated cation channels. Science 330: 55-60, 2010.

44. Lenzo JC, Turner AL, Cook AD, et al: Control of macrophage lineage populations by CSF-1 receptor and GM-CSF in homeostasis and inflammation. Immunol Cell Biol: July 5, 2011 (E-pub ahead of print) doi: 10.1038/icb.2011.58.
45. Fantl WJ, Johnson DE and Williams LT: Signalling by receptor tyrosine kinases. Annu Rev Biochem 62: 453-481, 1993.

46. Lu M and Krauss RS: N-cadherin ligation, but not Sonic hedgehog binding, initiates Cdo-dependent p38alpha/beta MAPK signaling in skeletal myoblasts. Proc Natl Acad Sci USA 107: 4212-4217, 2010.

47. Kang YJ, Chen J, Otsuka M, et al: Macrophage deletion of p38alpha partially impairs lipopolysaccharide-induced cellular activation. J Immunol 180: 5075-5082, 2008.

48. Wagner EF and Nebreda AR: Signal integration by JNK and p38 MAPK pathways in cancer development. Nat Rev Cancer 9: 537-549, 2009.

49. Thornton TM and Rincon M: Non-classical p38 map kinase functions: cell cycle checkpoints and survival. Int J Biol Sci 5: 44-51, 2009.

50. Karahashi H, Nagata K, Ishii K and Amano F: A selective inhibitor of p38 MAP kinase, SB202190, induced apoptotic cell death of a lipopolysaccharide-treated macrophage-like cell line, J774.1. Biochim Biophys Acta 1502: 207-223, 2000.

51. Elenitoba-Johnson KS, Jenson SD, Abbott RT, et al: Involvement of multiple signaling pathways in follicular lymphoma transformation: p38-mitogen-activated protein kinase as a target for therapy. Proc Natl Acad Sci USA 100: 7259-7264, 2003. 